\title{
GESTIÓN ADMINISTRATIVA DEPORTIVA \\ Caso de estudio: Liga Mayor de Fútbol Salvadoreña
}

Por: Licenciado Diego Tovar

Master en Dirección de Empresas con especialización en Mercadeo. Catedrático investigador, Universidad Centroamericana "José Simeón Cañas" dtovar@uca.edu.sv

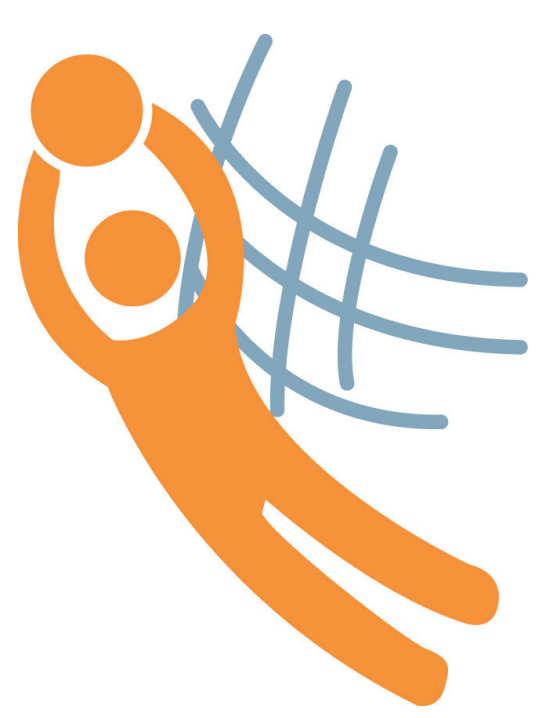

\section{RESUMEN}

"El deporte es demasiado juego para ser negocio y demasiado negocio para ser un juego", esta es una frase que se extrae del libro "Principles and practice of Sport Management" (Masteralexis, Barr y Hums), y que sin duda describe de una manera muy exacta le realidad no solo del fútbol, sino que del deporte en general.

En este artículo se presenta la situación actual del fútbol salvadoreño, desde una óptica administrativa-deportiva, con el objetivo de identificar oportunidades y áreas deficitarias, y de esta manera apuntar a investigaciones más profundas en el corto plazo. Para la realización de este articulo se ha tomado como base investigaciones realizadas a través del aula, trabajos de graduación, textos relacionados al deporte y recientes investigaciones desarrolladas por medios de comunicación salvadoreños.

Palabras claves: Fútbol, marketing deportivo, administración deportiva, patrocinio, liga mayor fútbol salvadoreño.

\section{INTRODUCCIÓN}

\section{La estructura del fútbol salvadoreño}

El fútbol salvadoreño, ha venido de más a menos en la última década, tocando su punto más bajo en el año 2013 cuando más de 15 jugadores profesionales fueron descubiertos por "amañar resultados" $* 1$ de juegos camino al mundial del 2014. Esto fue un duro golpe no solo para la selección mayor de fútbol, sino que para toda la estructura conformada por la primera, segunda y tercera división de fútbol "profesional" y las estructuras departamentales llamadas ADFAS, que vio como aficionados, patrocinadores y otros aliados estratégicos dejaron de apoyar estos proyectos, generando una crisis principalmente en los equipos de la primera división de fútbol, de los cuáles estudiamos la estructura administrativa y su gestión, encontrando muchas deficiencias en la parte de la gestión deportiva - administrativa.

Según datos proporcionados por la Primera División de Fútbol Salvadoreño (2018) durante el torneo Apertura 2018 (torneo que se juega de Julio a Diciembre) en total fueron 216,388 personas que asistieron a los diferentes escenarios deportivos, podríamos decir que apenas el $3.60 \%$ de nuestra población asiste al estadio versus Costa Rica en donde según fuentes de Diario 1 (2016) el 9.27\% de la población asiste a estos escenarios deportivos a presenciar juegos de los equipo de la Primera División del fútbol costarricense.

Para conocer un poco sobre la manera en la cual los equipos deportivos están organizados se realizó una investigación con estudiantes de la materia de investigación de mercados de la UCA durante el segundo semestre de 2018, tomando como base los 12 equipos de la Primera División en ese torneo, de acuerdo a la investigación el $66 \%$ de los equipos contaban con una Junta Directiva estructurada, mientras el restante $38 \%$ mencionaron que son una o dos personas las que usualmente se encargan de tomar las decisiones y administrar el equipo. 


\section{Gestión administrativa-deportiva.}

Todos los equipos cuentan con un pequeño grupo de encargados de la parte administrativa y logística, pero actualmente en el deporte profesional, la figura del "sport marketer", quien es el encargado de la comercialización y el marketing del equipo se vuelve cada vez más importante, así como el contar con un encargado o un área de comunicaciones dentro de los clubes. A finales del 2018, según recopilación de la información proporcionada por presidentes y representantes de equipos, no indicaron que un aproximado de 61 personas se dividían estas labores en los 12 equipos de la primera división de fútbol salvadoreño. Sin embargo únicamente Alianza F.C. mencionó tener bajo contrato permanente a parte de su equipo, mientras que los restante 11 equipos trabajan con jóvenes estudiantes en su mayoría universitarios, quienes trabajan ad honoren, recibiendo únicamente la oportunidad de acceder a los escenarios deportivos de manera gratuita y en algunos casos se entrega a estos un pequeño reconocimiento económico por el trabajo realizado, cabe resaltar también que sobre todo en el área de mercadeo y comunicaciones, buena parte del equipo (cámaras, computadoras, etc.) pertenece a los voluntarios.
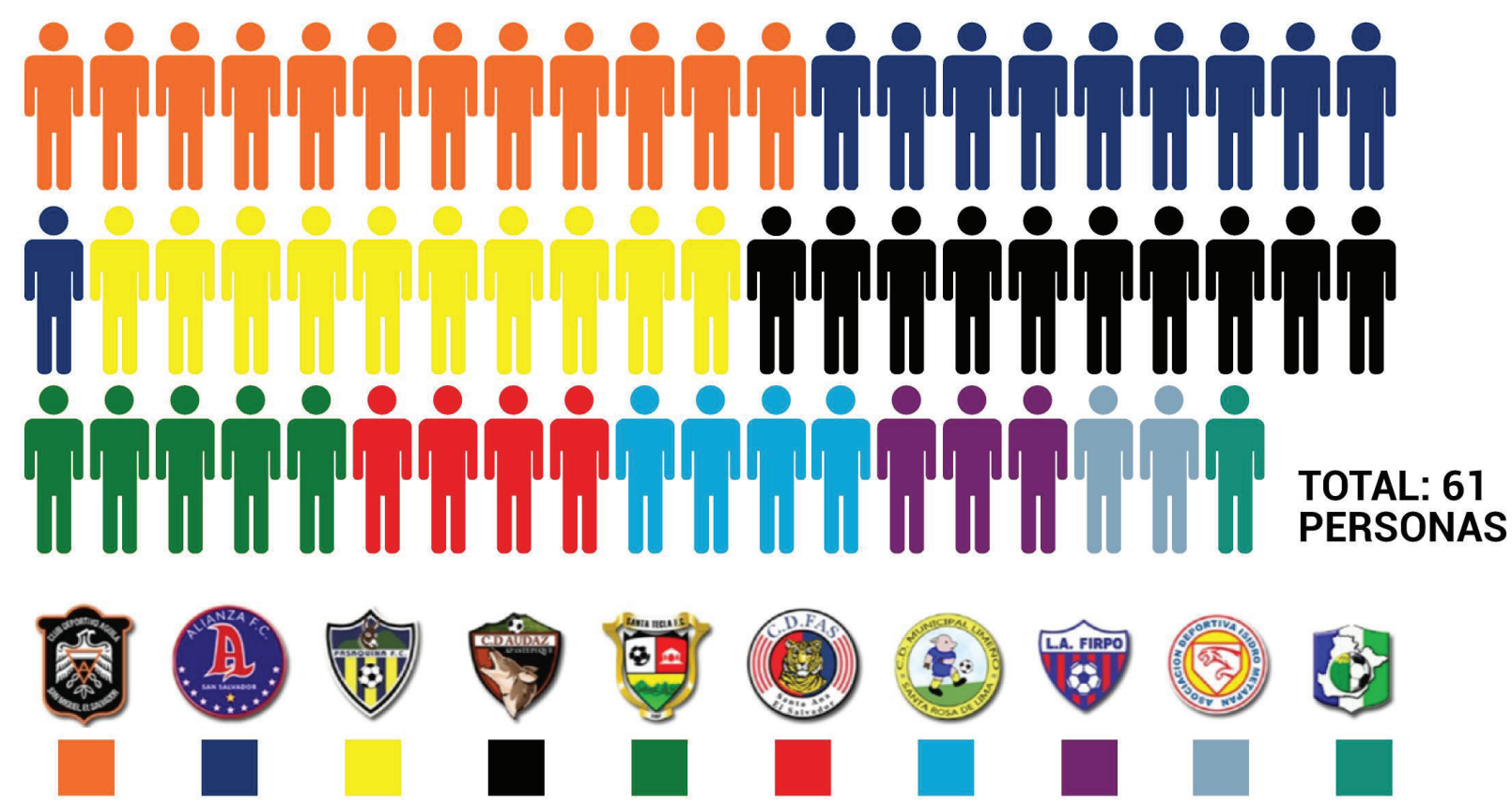

El tema de la planificación, uno de los pilares de la administración, es uno de los principales problemas en estas instituciones deportivas, según entrevistas a presidentes y representantes de los diferentes clubes.

La falta de recurso económico y de tiempo por parte de los directivos o dueños de equipos lleva a una gestión a través de la cual, la mayoría de los equipos van sobreviviendo el día a día, y parten únicamente de la elaboración de un presupuesto torneo a torneo, basado en los posibles patrocinadores y tomando en cuenta las taquillas recaudadas de torneo anterior, viáticos por participación en torneos internaciona-

les y derechos televisivos. Esta práctica es algo que mencionan en común el $100 \%$ de los entrevistados y que se convierte en la "planificación" torneo a torneo, agudizándose en algunos momentos por bajas taquillas o por retraso en los desembolsos pago por parte de patrocinadores. Esto genera que torneo a torneo se tengan retraso en pagos a jugadores y cuerpo técnico, que causa noticias negativas alrededor del club, que afectan la imagen de la marca, y volviendo más difícil el trabajo de comercialización, la invitación a aficionados al estadio, búsqueda de patrocinadores y posicionamiento de las marcas. 


\section{Los gobiernos municipales y el fútbol}

Otro aliado muy importante para 10 de los 12 equipos a excepción de L.A. Firpo que cuenta con su propio estadio y Alianza F.C. que alquila un estadio privado, es el apoyo de la municipalidad quién cede a los equipos la administración y mantenimiento de los estadios, haciéndolo cada alcaldía de manera diferente.Por ejemplo para el caso de Santa Tecla F.C. y Sonsonate, la municipalidad apoya con mantenimiento de la cancha, pago de iluminación en juegos nocturnos y en el caso de Santa Tecla la venta de espacios en vallas al interior del estadio. Mientras que para equipos como C.D. FAS, la alcaldía presta el inmueble para la realización de juegos, colaborando de manera compartida el mantenimiento de escenarios, pero no existe un apoyo para pago de iluminación en juegos nocturnos; para este año 2019, y como parte de requisitos solicitados por el tema de licencia de clubes, alcaldías como la de San Vicente, Santa Rosa de Lima, Chalatenango han hecho inversiones en los escenarios deportivos para que los equipos de esas ciudades puedan jugar sus partidos de local en sus sedes originales, esto tambien ha generado una buena imagen de los gobiernos locales con la población de la zona.

\section{El deporte necesita Marketing}

La falta de formación y de cultura deportiva- administrativa, así como el poco manejo en pro de posicionar y reforzar las marcas convierten los juegos de domingo a domingo en un producto poco o nada atractivo para el consumidor. Sin embargo el fútbol nacional, principalmente los equipos más populares como Alianza, C.D. FAS, C.D. Águila, tienen la oportunidad de explotar el tema de la tradición familiar respecto a seguir a un equipo o la identidad que representa respecto a la ciudad de donde proviene el equipo, tomando en consideración que en un $49 \%$ y $33 \%$ respectivamente estas son las razones por las cuales los aficionados siguen a sus equipos. Pero a pesar de esto, las promociones y comunicación generada en redes sociales, estadios y otros espacios no hace énfasis en estos aspectos, dejando de lado una gran oportunidad.

El tercer aspecto por el cual los aficionados se identifican con su equipo es por ser "un equipo ganador" en un $28 \%$ esto ha sido algo que ha aprovechado en lo últimos años Santa Tecla F.C. y Alianza F.C. que han aprovechado sus buenos momentos para mejorar su imagen y lograr en el caso del equipo verde obtener más seguidores y con Alianza F.C. reforzar su imagen $y$ hacer volver a sus aficionados al estadio.

Otro elemento que afecta a los equipos en cuanto asistencia de aficionados a sus juegos es la situación actual de muchos escenarios deportivos. En El Salvador en la época de los 90's equipos tradicionales como C.D. FAS o C.D. Águila, jugaban sus juego en horario de sábado por la noche, siendo este horario en la zona occidental el preferido por los seguidores en un $58 \%$, mientras en un $50 \%$ en la zona oriental, lastimosamente ninguno de estos escenarios deportivos cuenta con un sistema de iluminación en este momento, y esto ha hecho que la asistencia a estos escenarios disminuya, sumando aspectos como el clima, por ejemplo jugar un domingo a las 3:00 p.m., en un escenario como Santa Rosa de Lima, con temperaturas de $30^{\circ}$ a $40^{\circ}$ vuelven la experiencia de un juego de fútbol en una verdadera pesadilla y el aficionado prefiere no asistir y verlo por la televisión; por último falta de baños, aficionados violentos, accesos a personas de avanzada edad, ausencia de un parqueo seguro desaniman a los seguidores a compartir con sus equipos. 


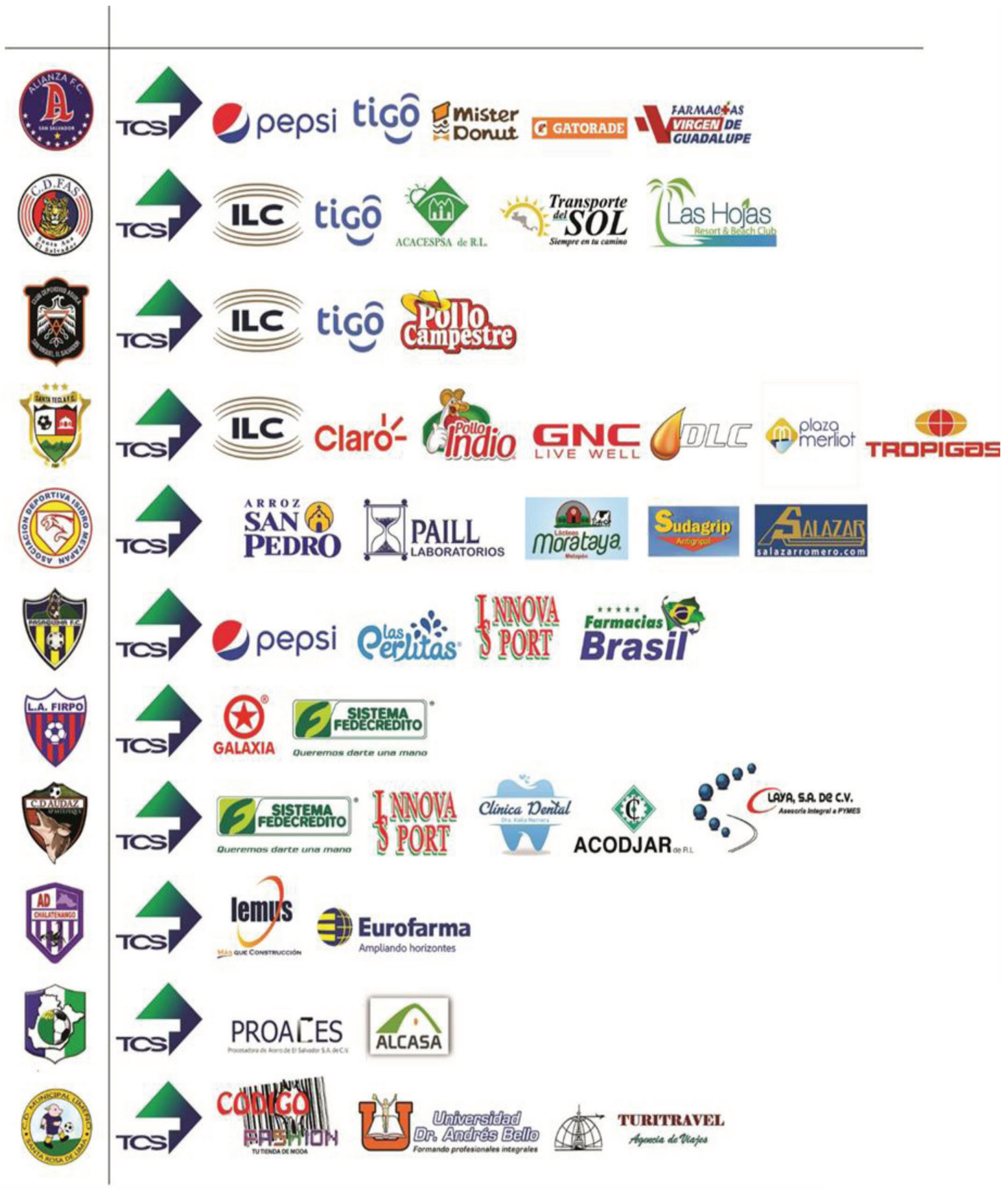

Imagen 2. Patrocinadores de los equipos de la primera división de fútbol salvadoreña
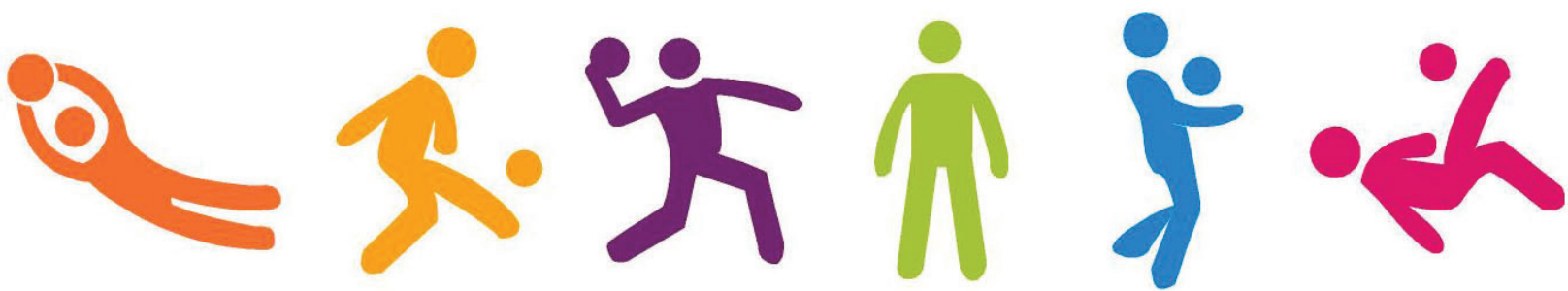
A pesar de verdaderas propuestas "ganar-ganar" planteadas por los clubes deportivos a distintas marcas comerciales, existen algunas entidades que apoyan el deporte, siendo TIGO la marca que un $36 \%$ de los aficionados asocian al fútbol salvadoreño, seguido por un $25 \%$ por PEPSI y un $22 \%$ por Pilsener. Estas marcas aparecen en los uniformes de juego de los equipos y en vallas dentro del estadio, siendo muchas veces el principal beneficio para estas marcas el "product placement" que se genera a través de los medios de comunicación y redes sociales durante la transmisión de juegos, noticias deportivas en televisión y prensa o el resumen de los mejores momentos de los partidos a través de youtube o noticieros.

\section{Conclusiones}

En conclusión, podemos observar que se tiene poco interés en modernizar la manera de administrar los clubes deportivos. Esto viene en parte por la falta de presupuesto, formación y un ente rector que apoye y exija la profesionalización del deporte, aunque a partir del 2019 una de las principales exigencias para los clubes de primera división es contar con licencia de clubes por parte de FIFA.

Siendo el fútbol el deporte más importante para los salvadoreños, se debe de pensar en explotar el mercado nostálgico en países como Estados Unidos e Italia, en donde existe una alta comunidad de salvadoreños, haciendo uso de herramientas como e-commerce o redes sociales, estos podrían generar ingresos a través de la venta de souvernir o abonados al club.
El fútbol en el país, como en cualquier parte del mundo, depende en gran manera del aporte de patrocinadores. En base a la investigación se puede concluir que para los equipos salvadoreños la búsqueda de patrocinadores supone un reto, ya que las empresas locales e internacionales ven poco atractivo el retorno que pueden obtener invirtiendo en ellos. Por lo cual, es muy importante administrar a los equipos de fútbol como verdaderas empresas, definiendo planes de mercadeo a las necesidades de la institución.

Un aspecto que muchos equipos han mejorado de a poco es la gestión de las redes sociales, la mayoría de sus cuentas están inactivas, se interactúa muy poco con los aficionados y no se plantean planes o metas para lograr mejores resultados por este medio, tomando en cuenta que las redes sociales ahora son uno de los medios de comunicación más eficientes y económicos. Por lo cual, la capacitación en el área de marketing digital con estrategias concretas debe de ser algo que los equipo deben trabajar e invertir.

Se recomienda por último fortalecer la marca "Primera División de Fútbol Salvadoreño", trabajando de manera conjunta con los diferentes clubes de fútbol, que puedan elevar el prestigio del torneo, atrayendo así a más inversionistas nacionales e internacionales y de esta manera mejorar la competitividad de los equipos.

\section{Bibliografia:}

Esteve Calzada; Show me the money "cómo conseguir dinero a través del marketing deportivo

Ascencio M., Barrera J., Figueroa C., González J., UCA, Trabajo de graduación:

Diagnóstico de la gestión deportiva por parte de los clubes de la primera división del fútbol salvadoreño, al año 2018.
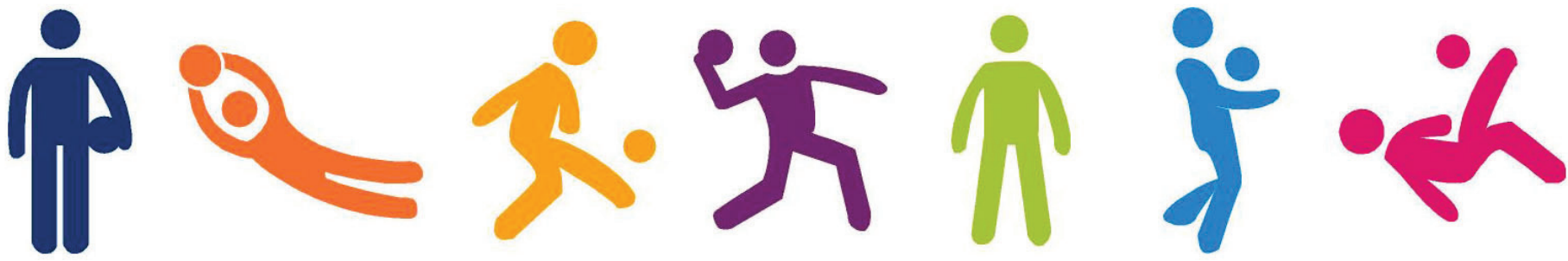